\title{
AVALIAÇÃO DA QUALIDADE DE VIDA EM ID OSOS SUBMETIDOS AO TRATAMENTO HEMODIALÍTICO
}

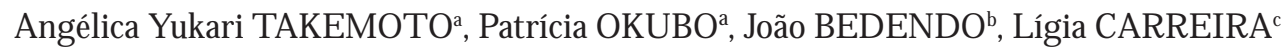

\section{RESUM 0}

A hemodiálise afeta não só aspectos físicos, como psicológicos e sociais, com repercussão na vida pessoal e familiar. Considerando que no Brasil está ocorrendo um aumento da população idosa, esta pesquisa objetivou avaliar a qual idade de vida dos idosos com insuficiência renal crônica, submetidos ao tratamento hemodialítico. T rata-se de uma pesquisa quantitativa, de caráter descritivo exploratório, com idosos de um serviço especializado em hemodiálise de Guarapuava, Paraná. Os dados foram coletados entre maio a junho de 2010, através de um instrumento estruturado e, após, submetidos ao programa estatístico Statistica 7.1. A nal isando os domínios do questionário, 0 maior escore diz respeito ao domínio social $(70,42)$ e o menor, ao domínio físico $(49,37)$. A ssim, a qualidade de vida desses idosos apresentou-se baixa, com variações de acordo com o domínio anal isado. Pesquisas voltadas à avaliação de qualidade de vida são relevantes e instrumentalizam a prática diária do cuidado.

D escritores: Cuidados de enfer magem. I doso. Insuficiência renal crônica. Diálise renal. Qualidade de vida.

\section{RESUMEN}

H emodiálisis afecta aspectos física y psicológica y social, con repercusiones en la vida personal y familiar. M ientras que en B rasil está experimentando un aumento de la población de edad avanzada, este estudio tuvo como objetivo eval uar la calidad devida de los pacientes ancianos con insuficiencia renal crónica sometidos a hemodiálisis. Setrata de un estudio cuantitativo, exploratorio descriptivo con pacientes ancianos en un centro especializado en hemodiálisis $G$ uarapuava, Paraná, B rasil. L os datos fueron recogidos entre mayo y junio de 2010 a través de un instrumento estr ucturado y, después someterse el programa estadístico Statistica 7.1. A nalizar los dominios del cuestionario, la puntuación más alta con respecto al ámbito social $(70,42)$ y menor dominio físico $(49,37)$. L o tanto, la calidad de vida de estos ancianos tenían ser bajo, con variaciones según ámbito analizado. L a investigación destinada a evaluar la calidad de vida son relevantes e instrumentalizar la práctica diaria de atención.

D escriptores: A tención de enfermería. Anciano. Insuficiencia renal crónica. D iálisis renal. Calidad de vida. T ítulo: E valuación de la calidad de vida en personas mayores sometidos a hemodiálisis.

\section{ABST RACT}

H emodialysis affects not only physical but psychological and social aspects, with repercussions on personal and family life. Considering the increase in the elderly population in B razil, this study aims to evaluate the quality of life of elderly patients with chronic renal failure under going hemodialysis. T his is a quantitative, descriptive explorator y study with el derly patients held in a facility specializing in hemodialysis in G uarapuava, Paraná, B razil. T he data were collected betw een $M$ ay and J une 2010, through a structured instrument and were submitted to the statistical software Statistica 7.1. Analyzing the domains of the questionnaire the highest score refers to the social domain (70.42) and the low est, to the physical domain (49.37). T hus, the quality of life of these elderly had to be low, with variations according to the analyzed field. R esearches aimed at assessing quality of life are relevant and instrumentalize the daily practice of care

D escriptors: N ursing care. Aged. R enal insufficiency, chronic. Renal dialysis. Quality of life.

Title: E valuation of quality of life in elders undergoing hemodialysis.

\footnotetext{
a E nfer meira, M estranda em E nfer magem pelo Prog rama de Pós-G raduação em E nfer magem da U niver sidade E stadual de M aringá (UE M ),

M aringá, Paraná, Brasil.

- D outor em Enfermagem, D ocente A djunto da Pós-G raduação em Enfer magem da UEM , M aringá, Paraná, Brasil.

‘ D outora em Enfer magem, D ocente A djunta da G raduação e da Pós-G raduação em Enfermagem da U E M, M aringá, Paraná, Brasil.
} 


\section{INT RODUÇÃO}

A D oença Renal Crônica (D RC) refere-se à destruição progressiva gradual e irreversível de grande número de néfrons e consequentemente da função renal ${ }^{(1)}$. A Sociedade Brasileira de N efrologia, estima que aproximadamente 2 milhões de brasileiros são portadores de DRC e aproximadamente $70 \%$ não sabem disso, e 70 mil estão em tratamento por diálise e 25 mil com transplantes renais com enxerto funcionante. As três principais causa de D RC no mundo são Diabetes mellitus, Hipertensão A rterial e G lomerulonefrites ${ }^{(2)}$.

Dentre os tratamentos disponíveis os quais substituem parcialmente a função renal encontra-se diálise peritoneal e suas modalidades, transplante renal e hemodiálise, escolhida conforme as condições clínicas e psicológicas ${ }^{(3)}$.

A hemodiálise, uma das opções para o tratamento da insuficiência renal crônica, consiste na filtração extracorpórea do sangue por intermédio de uma máquina provocando uma série de circunstâncias para o portador de DRC, afetando não só aspectos físicos, como psicológicos e sociais, e com repercussão na vida pessoal e familiar ${ }^{(4)}$. T rata-se de um processo de filtragem e depuração do sangue de substancias tóxicas como uréia e creatinina por meio de um filtro de hemodiálise ou capilar devido a deficiência do mecanismo no organismo do paciente com $D R C^{(1)}$. E sse processo contínuo leva a conflitos psicossociais, como alter ação da imagem corporal, dependência, perspectiva de uma morte potencial, restrições dietéticas e hídricas e alterações na interação social não só do paciente, como também da sua família ${ }^{(3,5,6)}$.

D essa forma, vê-se a necessidade de adaptação familiar ao tratamento, exigindo compromisso e dedicação, sendo essas intensas e tendem a aumentar com a evolução da doença ${ }^{(7)}$. N esta adaptação à nova rotina, pacientes e familiares precisam absorver informações, indicações e prescrições, desencadeando ansiedade cansaço e estresse, alterando, portanto, sua qual idade de vida(6).

A qualidade de vida trata-se de um forte indicador de avaliação dos atendimentos prestados pelos serviços de saúde, aliando o processo saúdedoença com a efetividade dos procedimentos utilizados para o tratamento e reabilitação(8). N esse contexto, a avaliação da qualidade de vida de portadores de doenças crônicas tem se tornado alvo frequente 0 âmbito das pesquisas científicas, uma vez que essas geralmente estão relacionadas a um prognóstico negativo. 0 diagnóstico da patologia crônica exerce um impacto no cotidiano desses indivíduos, pela mudança e acréscimo de muitas tarefas, como as transformações das relações sociais, o tratamento a ser seguido, as possíveis al terações na aparência pessoal, entre outros aspectos ${ }^{(7)}$.

A lém disso, essas pessoas estão limitadas por uma doença incurável, passíveis de muitas complicações e em constante exposição de situações estressoras como a sobrevivência dependente de uma máquina de diálise, a frequente permanência no ambiente hospitalar e mudanças na dieta alimen$\operatorname{tar}^{(9)}$.

Com a inver são da pirâmide etária e o consequente envelhecimento populacional, a influência de doenças crônicas não-transmissíveis (DCNT), como a hipertensão arterial e o diabetes mellitus, é considerado fator determinante para o desenvolvimento da insuficiência renal crônica, justificando 0 aumento crescente de indivíduos em tratamento dialítico ${ }^{(10)}$. Sabe-se que a qualidade de vida de pacientes com DRC apontam que parte dessa população está entre 40 e 80 anos de idade, mostrando a importância da avaliação da qualidade de vida nessa faixa etáriaa ${ }^{(3,6)}$. Aliado a essa complexa situação, é importante acrescentar as dificuldades impostas pelo avançar da idade.

N esse sentido, é importante realizar uma avaliação da qualidade de vida dos idosos submetidos à terapia de tratamento hemodialítico, através do qual se pretende fornecer subsídios para que a equipe de saúde tenha uma percepção quanto ao impacto dessa condição crônica na vida dos idosos, contribuindo para gerar mudanças condizentes com a realidade e possibilitando uma atuação profissional adequada frente a essa população. D essa forma, o objetivo do estudo foi avaliar a qualidade de vida dos idosos com insuficiência renal crônica, submetidos ao tratamento de hemodiálise.

\section{MÉTODOS}

T rata-se de um estudo descritivo e exploratório, de caráter quantitativo, realizado com idosos usuários de um serviço de hemodiálise do município de Guarapuava, Paraná. A tualmente, sua área de abrangência, a 5a Regional de Saúde, possui cadastrados 697 estabelecimentos de saúde. Desses, o município de G uarapuava, contribui com 39 unidades básicas de saúde, cinco hospitais gerais, cin- 
co postos de saúde, nove policlínicas, 202 consultórios isolados e 45 clínicas especializadas, incluindo a instituição para pessoas dependentes de hemodiálise ${ }^{(11)}$.

Essa clínica presta atendimentos aos pacientes renais crônicos desde outubro de 1984 e a primeira hemodiálise realizada em janeiro de 1985, sendo a única clínica do município que atende pessoas em tratamento dialítico nesta região, abrangendo o Sistema Ú nico de Saúde e outros convênios de saúde ${ }^{(12)}$, atendendo atualmente 144 pacientes distribuídos em dias alternados de segunda à sábado, entre os turnos da manhã, tarde e noite. Desse total de atendimentos, 138 indivíduos são da modalidade de hemodiálise.

A população participante da investigação foi composta por idosos com 60 anos e mais, diagnosticados por DRC, em tratamento no referido serviço de hemodiálise e de ambos os sexos, critérios estes que compôs um total de 48 idosos. Com a exclusão de dois idosos que apresentaram instabilidade clínica e uma com dificuldade de compreensão, além de dois indivíduos que se recusaram em participar do estudo, e uma que estava de alta das sessões de hemodiálise e dois que haviam falecidos, foram obtidos 40 formulários.

A coleta de dados foi realizada entre maio a junho de 2010, através da aplicação de um instrumento constituído por duas partes, sendo a primeira parte referente as questões sócio-demográficas e de saúde e a segunda abordando a avaliação da qualidade de vida, com a incorporação do questionário abreviado de Qualidade de Vida da Organização M undial da Saúde (W HOQOL-brief). 0 WHOQOL-brief é composto por 26 perguntas, divididas em quatro domínios: F ísico, Psicológico, Social e do M eio A mbiente, e seu resultado é obtido através de um escore analógico, variando de 0 a 100, no qual quanto maior o escore, maior a qualidade de vida, e quanto menor o escore, menor a qualidade de vida ${ }^{(13)}$. As informações foram tabuladas e analisadas em planilhas do tipo E xcel ${ }^{\circledR}$ e posteriormente transferidas para 0 softw are Statistica 7.1, aplicando a análise descritiva e frequência simples.

Essa pesquisa foi realizada mediante autorização da direção clínica do serviço hemodialítico e a devida aprovação do Comitê de Ética em Pesquisa (COPEP) da U niversidade Estadual de Maringá (UEM ), por meio do parecer $n=220 / 2010$, obedecendo-se aos aspectos éticos estabelecidos pela
Resolução 196/ 96 do Conselho Nacional de Saúde $^{(14)}$. Os pacientes foram abordados na própria clínica, durante a sessão de hemodiálise. I nicialmente, foi realizado o esclarecimento da pesquisa ao idoso e, logo após, foi solicitado a assinatura do termo de consentimento livre e esclar ecido caso 0 mesmo aceitasse a participar do estudo. A pós 0 aceite na participação foi aplicado os dois instrumentos para obtenção das informações pertinentes ao objeto do estudo.

\section{RESULT ADOS E DISCUSSÃO}

\section{Caracterização dos participantes}

Da amostra de 40 idosos, 29 (72,5\%) foram homens e 11 (27,5\%) mulheres, com idade média de 68,7 anos, com variação entre 6,5 anos (61-83) anos.

Ao analisarmos a situação conjugal, 25 $(62,5 \%)$ dos idosos são casados, dez $(25,0 \%)$ viúvos, dois $(5,0 \%)$ em união estável, dois $(5,0 \%)$ separados e um idoso informou ser solteiro (2,5\%). I dentificamos que $16(40,0 \%)$ residiam com o companheiro e filhos, $11(27,5 \%)$ com o companheiro e seis $(15,0 \%)$ somente com os filhos; os demais entrevistados residiam com a empregada ( $\mathrm{N}=1$; $2,5 \%)$, sobrinha ( $N=2 ; 5,0 \%)$, irmão $(\mathrm{N}=1 ; 2,5 \%)$ ou ainda moravam sozinhos ( $\mathrm{N}=3 ; 7,5 \%)$.

Quanto ao nível de escolaridade, 17 (42,5\%) idosos referiram o ensino fundamental incompleto, destacando para a presença de seis $(15,0 \%)$ indivíduos que nunca estudaram e quatro $(10,0 \%)$ com o ensino superior completo. Quanto à renda familiar $34(85,0 \%)$ encontra-se com um a três salários mínimos. U m idoso (2,5\%) referiu renda inferior a um salário e cinco idosos $(12,5 \%)$ relataram renda superior a três salários mínimos. Essa baixa renda mensal se justifica aos meios de aquisição financeira, como a falta de um vínculo empregatício formal, aposentadorias e auxílio-doença.

E ntre pacientes dependentes da hemodiálise, considerando os aspectos físicos, sociais e emocionais, $11,8 \%$ nunca estudaram e não sabem ler nem escrever e $29,4 \%$ possuíam o ensino fundamental incompleto ${ }^{(15)}$. E sses dados devem ser considerados significantes para os profissionais de saúde no que tange a comunicação com essas pessoas, pois se necessita utilizar uma linguagem acessível para garantir 0 entendimento, principal mente, quando se trata de orientação e ou educação em saúde para a prevenção de complicações referentes ao tratamento. 
T rinta e cinco $(87,5 \%)$ dos entrevistados já estavam aposentados, e cinco (12,5\%) ainda estavam trabalhando. L evando em consideração apenas os aposentados, a idade média de estagnação do trabalho foi de 46,7 $\pm 24,3$ anos. No entanto, havia idosos que apesar de aposentados ainda continuavam trabalhando e dentre as ocupações anteriores, a que se destaca é o trabal ho na lavoura, referido por $11(31,5 \%)$ participantes, seguido dos nove $(25,7 \%)$ idosos com a profissão de serviços gerais, seis $(17,1 \%)$ servidores públicos, quatro $(11,4 \%)$ trabal hadores autônomos, quatro (11,4\%) comerciantes e um $(2,9 \%)$ idoso que não estava trabalhando antes da aposentadoria. É importante ressaltar que quando indagados sobre essa questão, a maior parte dos idosos demonstrou sentimentos de incapacidade, uma vez que desejavam estar trabalhando no momento.

0 trabalho geralmente demonstra importância na qualidade de vida das pessoas com DRC, principal mente no que se refere a auto-realização profissional. A DRC e os seus tratamentos não estabelecem uma barreira direta à atividade laboral, porém causam limitações importantes, induzindo aos afastamentos e aposentadorias precoces devido a doença ${ }^{(7)}$. Pode haver comprometimento do trabaIho relacionado diretamente com o tempo da hemodiálise ${ }^{(3)}$.

A incapacidade física limitada para a realização das atividades diárias provoca também sentimentos que deprimem a qualidade de vida do ser humano, pois o trabalho é uma das formas pelo qual o indivíduo se expressa, se identifica e se realiza no mundo(7,16).

Quando questionado pela necessidade de cuidador, 29 (72,5\%) indivíduos relataram não precisar de auxílio e 11 (27,5\%) reconheceram a sua necessidade, destacando a ajuda na deambulação e alimentação. D entre os cuidadores foram citados 0 companheiro ( $N=5)$, filhos ( $N=3)$, familiares ( $N=$ 2) e a empregada ( $\mathrm{N}=1)$. N ota-se que a primeira rede de apoio social ao paciente com DRC é a família, uma vez que a maioria dos pacientes reside com o companheiro e/ ou filhos. Como a maior parte dos idosos possui um companheiro, esse certamente irá exercer o papel do cuidador. 0 papel do cuidador é fundamental desde o início do tratamento, a medida que ocorre a evolução da doença, o paciente passa a apresentar dificuldades físicas que o impedem de realizar de forma autônoma suas atividades, exigindo o compromisso e dedicação familiar, traduzida de diversas formas e em diferentes situações ${ }^{(7)}$.

Contudo, apesar de poucos idosos serem dependentes, é importante destacar o envolvimento e a participação da família e/ ou amigos na prestação dos cuidados ao paciente hemodialítico, principalmente a partir da realização de atividades de educação em saúde. Cabe ao profissional de saúde assumir uma abordagem de cunho participativo para suprir todas as dúvidas e medos que envolvem essa doença e seu tratamento ${ }^{(15)}$.

M uitas vezes os pacientes dialíticos mostramse inter essados em compartilhar suas experiências e necessidades, retratados através das dificuldades impostas pelo dia-a-dia à pessoa renal crônica pelo distanciamento entre o saber popular e o saber científico advindo do conhecimento dos profissionais de saúde ${ }^{(17)}$.

A idade da descoberta da DRC foi em média de 62,3 \pm 9,4 (40-78) anos e o tempo médio de manutenção da hemodiálise da amostra foi de $42 \pm$ 41 (5-180) meses. A vivência de receber 0 diagnóstico de DRC revela-se, em diversas situações, como um dos momentos mais difíceis para o paciente e 0 meio familiar. São experimentados, entre outros sentimentos, a angústia diante do desconhecido e o medo frente à possibilidade do sofrimento e morte. Chama a atenção para a importância da fé para o enfrentamento dessa situação, no sentido de se tornar uma fonte de esperança e força a esses indivíduos(16).

Paralelamente a esse fator, 0 aumento no tempo do tratamento hemodialítico sugere maior interferência no aspecto emocional dos sujeitos e, consequentemente, um possível comprometimento das relações familiares e sociais. N esse sentido, é evidente uma ambivalência relacionada ao tratamento. Se por um Iado a hemodiálise Ihe permite uma ilusão de independência da doença durante 0 tempo entre uma sessão de diálise e outra, por outro lado, no momento em que o paciente está conectado a máquina, as limitações da doença e sua dependência do aparelho aparecem com toda a sua intensidade ${ }^{(16,18)}$.

\section{Avaliação da qualidade de vida}

Com relação a percepção de sua própria qualidade de vida, 20 (48,8\%) idosos atribuíram uma avaliação mediana, descrita no instrumento utilizado como "nem ruim e nem boa". Já no que con- 
cerne 0 quão satisfeitos estão com a sua saúde, foi referida satisfação por $29(70,7 \%)$ idosos. A hemodiálise prolonga a vida do doente, reduz o sofrimento e previne futuras complicações. Esse tratamento é responsável por um cotidiano monótono e restrito, e as atividades limitadas após o início do tratamento. No entanto, os indivíduos ainda reconhecem o tratamento hemodialítico como fator de sobrevivência e garantia da manutenção do bemestar ${ }^{(7,16)}$.

Com 0 avançar da idade e da doença renal, a percepção dos indivíduos quanto à sua qualidade de vida pode encontrar-se modificada pela característica de cronicidade da D RC e do tratamento, sendo comum a presença do conformismo quanto ao seu estado de saúde. 0 indivíduo com D RC teria a necessidade de ser "normal", ou seja, iguais aqueles não doentes e, dessa forma, atribuem satisfação com a própria saúde como forma de dizer: sou como os demais ${ }^{(15,19)}$.

A nalisando os domínios do W HOQOL-brief, o maior escore diz respeito ao domínio social, enquanto o domínio físico obteve o escore mais baixo, conforme a T abela 1.

T abela 1 - Distribuição dos escores do W HOQOL brief deidosos em tratamento hemodialítico. G uarapuava, PR, maio/ jun. 2010.

\begin{tabular}{lcc}
\hline D omínios & $\begin{array}{c}\text { Escores } \\
\text { Brutos }\end{array}$ & $\begin{array}{c}\text { Escores } \\
\text { Transformados } \\
\mathbf{0 - 1 0 0}\end{array}$ \\
\hline Físico & 20,85 & 49,46 \\
Psicológico & 19,72 & 57,18 \\
Social & 11,45 & 70,42 \\
M eio-Ambiente & 25,17 & 53,67 \\
\hline
\end{tabular}

Fonte: Serviço Especializado em H emodiálise. Guarapuava, PR.

Foram relatos constantes durante as entrevistas a falta de energia e a consequente presença da fadiga durante o dia, a dificuldade para dormir, a dependência de medicação e tratamentos e também as alterações quanto à capacidade para o trabalho, condições que são justificadas na redução do escore na categoria de dimensão física.

Os sinais de deterioração musculoesquelética e frequentes fraquezas ocasionadas pela hemodiálise são responsáveis por diversos sintomas desagradáveis, como mal-estar, náuseas, diarréia, dispnéia e tosse, comprometendo assim, o bem-estar físico do renal crônico ${ }^{(15)}$. I nerente a essa situação, com 0 avançar da idade, observa-se um maior com- prometimento nas atividades físicas e funcionais das pessoas ${ }^{(18)}$. Resultado semelhante foi encontrado em um estudo realizado em São Paulo, com a utilização do instrumento SF -36, no qual referem a qualidade de vida influenciada negativamente pelo estado geral de saúde do paciente ${ }^{(20)}$, nos per mitindo refletir que o escore baixo do domínio físico pode influenciar os demais domínios, como o domínio psicológico e, além disso, sugerindo que não se pode dispensar cuidados a um problema isolado, mesmo que seja uma manifestação física, esta deve ser tratada dentro do contexto de vida da pessoa.

Os pacientes submetidos ao tratamento hemodialítico, geralmente apresentam alterações de humor decorrente da sua disposição biológica que pode mudar repentinamente entre uma e outra sessão de tratamento. I sso porque o acúmulo de líquidos no organismo ou a retirada em excesso pelas sessões de diálise pode provocar a irritação, mauhumor e depressão(15).

Dentre as questões abordadas no domínio social estão a inclusão do indivíduo na sociedade em que vive e suas relações. Sabe-se que problemas referentes a relação social em indivíduos com doença renal crônica são a timidez e sofrimento diante de atitudes preconceituosas ${ }^{(17)}$. No entanto, o domínio social tem sido indicado neste e em outros estudos de qualidade de vida como um dos mais altos. $N$ este estudo foi referido um fortalecimento da rede de apoio familiar e social, particularmente após a descoberta da DRC, foram recorrentes nos discursos.

0 apoio de familiares e amigos nesse processo, proporcionando força e coragem para a continuação do tratamento. A sensação de não estar sozinho e de sentir-se apoiado por outras pessoas merece destaque $^{(16)}$. Os idosos com DCNT desenvolvem maiores dependências, pois enfrentam mudanças sociais, econômicas, físicas e emocionais. Por sua vez, necessitam e possuem a presença e apoio de familiares e/ ou amigos para o cuidado. Esse envolvimento familiar é indispensável para o suporte ao idoso com DRC em tratamento hemodialíti$\mathrm{CO}^{(19)}$.

A partir do enfrentamento da cronicidade da doença é possível compreender o significado e refletir sua influência no cotidiano do paciente, inclusive com relação ao meio em que vive ${ }^{(16)}$. Q uando indagados sobre as questões ambientais, grande parte dos idosos relatou a preocupação financeira, o incômodo com ruídos, a facilidade de aces- 
so ao serviço de saúde, a ausência de participação em locais de recreação elazer e a fal ta de prática de exercícios físicos.

A indisposição física em realizar caminhadas e conhecer locais de entretenimento pode ter relação com vários fatores, como a idade avançada, a falta de hábitos em realizar essas atividades e as alterações eletrolíticas advindas da própria condição da doença que podem gerar cansaço físico. T ratando-se de pessoas com D RC em tratamento hemodialítico o fator fadiga pode ser considerado determinante nesse aspecto ${ }^{(15)}$.

N o entanto, poucos são estudos encontrados relacionando as questões do meio ambiente com a melhora ou piora da qualidade de vida do paciente renal crônico. F ato este rel evante e que pode ser objeto de futuras investigações, uma vez que são aspectos que possuem influência direta na qualidade de vida, como confirmado pelo escore obtido na presente pesquisa.

A partir dessa pesquisa, fica evidente que a qualidade de vida é definida pela interação de fatores diversos como tempo de diagnóstico, terapêutica, estado clínico geral e rede social de suporte, explicando a discordância dos achados pela diversidade de possibilidades de inter ações entre estes fatores ${ }^{(18)}$. A importância da percepção que cada paciente tem de sua vida, saúde e doença, considerando seu ponto de vista acerca da solução dos seus problemas, trazem à tona o desenvol vimento de um trabal ho voltado não só a doença, mas ao doente e priorizando a suas interações sociais, familiares e com a equipe de saúde ${ }^{(16)}$.

Assim, percebe-se a necessidade de intervenções pelo conhecimento das reais necessidades dos pacientes hemodialíticos. Essa pesquisa fornece elementos importantes para a execução de novas estratégias a fim de promover a melhoria na qualidade de vida desses idosos, através da otimização do tratamento hemodialítico e de proporcionar um cuidado de enfermagem mais qualificado a essa população, além de servir como incentivo para novas pesquisas.

A o enfermeiro, exclusivamente, compete a utilização do processo de enfermagem, com o objetivo de aprimorar sua qualidade de trabal ho e consequentemente melhorar sua assistência, uma vez que esse instrumento permite a identificação das necessidades de saúde do indivíduo que precisam de intervenção.

\section{CONSIDERAÇÕES FINAIS}

A qualidade de vida de indivíduos idosos portadores de DCNT é fundamental, principalmente no que diz respeito ao impacto da doença em suas vidas e o processo de adaptação à patologia, por se tratar de um processo demorado esofrido, ora para si próprio, ora para sua família.

O instrumento WHOQOL-brief mostrou aptidão para avaliar a qualidade de vida da população em estudo, uma vez que corroborou para o alcance dos objetivos, além de oferecer elementos importantes para auxiliar na prestação de uma assistência de enfermagem mais qualificada e humanizada, de acordo com as suas necessidades de saúde.

Os idosos submetidos a tratamento hemodialítico apresentaram uma qualidade de vida baixa, com variações de acordo com os domínios analisados. Por se tratar de indivíduos com uma doença crônica, progressiva e irreversível identificouse que o escore mais prejudicado trata-se do domínio físico, o qual aborda questões relativas ao estado de saúde do paciente.

E m contrapartida, a existência de boas relações no âmbito social, principalmente com familiares, influencia para o aumento no escore do domínio social. É importante destacar para a boa percepção individual da qualidade de vida desses pacientes, sugerindo um avançado estágio de adaptação à patologia e ter apêutica utilizada.

A o profissional de enfermagem é essencial um olhar ampliado de seus clientes contemplando aspectos tanto do domínio físico, como psicológico, social e meio-ambiente para a compreensão das reais necessidades de cuidado desses indivíduos. T rata-se de um alicerce para manter uma boa saúde, a readequação das ações terapêuticas a partir da compreensão da vida habitual do paciente com D RC. A ssim, pesquisas voltadas à avaliação de qualidade de vida são rel evantes, uma vez que instrumentalizam a prática diária do cuidado.

\section{REFERÊ NCIAS}

$1 \mathrm{~N}$ ascimento $C D, M$ arques, IR . Intervenções de enfermagem nas complicações mais frequentes durante a sessão de hemodiál ise: revisão da literatura. R ev Bras Enferm. 2005;58(6):719-22.

2 U niver sidade F ederal de Rondônia. Sociedade Brasileira de $N$ efrologia e Curso de M edicina da U N IR promovem Campanha de Prevenção da D oença Renal Crô- 
nica [ Internet] . Porto Velho; 2010 [ citado 2010 M ar 29] . D isponível em: http:/ / www.rondonoticias.com. $\mathrm{br} /$ ?noticia,77283,sociedade-brasil eira-de nefrologiae-curso-de-medicina-da-unir-promovem-campanhade-preveno-da-doena-renal-crnica.

$3 \mathrm{M}$ artins M RI, Cesarino CB. Qualidade de vida de pessoas com doença renal crônica em tratamento hemodialítico. Rev Latino-A m E nfermagem. 2005;13(5):670-6.

4 Riella M C. Princípios de nefrologia e distúrbios hidroeletrolíticos. 4a ed. Rio de Janeiro: G uanabara K oogan; 2003.

5 Souza E F, M artino M M F, Lopes M H BM . Diagnósticos de enfermag em com tratamento hemodialítico utilizando o modelo de I mogene King. Rev Esc E nferm U SP. 2007;41(4):629-35.

6 Higa K, Kost MT, Soares DM, M orais M C, Polins BRG. Qualidade de vida de pacientes portadores de insuficiência renal crônica em tratamento de hemodiálise. A cta Paul E nferm. 2008;21(n esp):203-6.

7 Carreira L, M arcon SS. Cotidiano e trabalho: Concepções de indivíduos portadores de insuficiência renal crônica e seus familiares. Rev Latino-Am Enfermagem. 2003;11(6):823-31.

8 Cianciarullo TI, F ugulin F M T, Andreoni S. A hemodiálise em questão: opção pela qualidade assistencial. São Paulo: İ cone; 1998.

9 Caiuby AVS, L efèvre F, Pacheci-Silva A. A nálise do discurso dos doadores renais: abordagem da psicologia social. J Bras N efrol. 2004;26(3):137-44.

10 Romão J r J E, Pinto SW L, Canziani M E, Praxedes J N, Santello J L, M oreira J CM. Censo SBN 2002: informações epidemiológicas das unidades de diálise do Brasil. J Bras N efrol. 2003;25(4):188-99.

11 M inistério da Saúde (BR), D epartamento de Informática do Sistema Ú nico de Saúde, Cadastro N acional de Estabelecimentos de Saúde. E stabelecimentos de Saúde: Paraná [ Internet] . Brasília (DF ); 2010 [ citado 2010 out 19] . Disponível em: http:/ / tabnet. datasus.gov.br/ cgi/ tabcgi.exe?cnes/ cnv/ estabpr.def.

\section{E ndereço da autora / Dirección del autor / Author's address:}

Angélica Yukari T akemoto

Rua Dr. Saulo Porto Virmond, 884, bl. C, ap. 104, Jardim N ovo Horizonte

87005-090, M aringá, PR

E - mail: angelica.takemoto@ hotmail.com
12 A breu IS. Qualidade de vida relacionada à saúde de pacientes em hemodiálise no município de Guarapuava - PR [ dissertação] . R ibeirão Preto: E scola de E nfermagem, U niversidade de São Paulo; 2005.

13 F leck M PA, Leal OF, Louzada S, X avier M, Chachamovich $E$, Vieira $G$, et al. Desenvolvimento da versão em português do instrumento de avaliação de qualidade de vida da OM S (W HOQOL-100). Rev Bras Psiquiatr. 1999;21(1):19-28.

14 M inistério da Saúde (BR), Consel ho N acional de Saúde. Resolução 196, de 10 de outubro de 1996: diretrizes e normas regulamentadoras de pesquisa envolvendo seres humanos. Brasília (DF); 1996.

15 T rentini M , Corradi E M , Araldi M AR, T igrinho FC. Qualidade de vida de pessoas dependentes de hemodiálise considerando alguns aspectos físicos, sociais e emocionais. Texto Contexto Enferm. 2004;13(1): 74-82.

16 Pereira L P, G uedes M VC. H emodiálise: a percepção do portador renal crônico. Cogitare E nferm. 2009;14 (4):689-95.

17 Queiroz M VO, D antas M CQ, Ramos IC, Jorge M SB. Tecnologia do cuidado ao paciente renal crônico: enfoque educativo-terapêutico a partir das necessidades dos sujeitos. T exto Contexto E nferm. 2008;17 (1):55-63.

18 Castro M , Caiuby AVS, Draibe SA, Canziani M AF. Qualidade de vida de pacientes com insuficiência renal crônica em hemodiál ise avaliada através do instrumento genérico SF -36. Rev A ssoc M ed Bras. 2003; 49(3):245-9.

19 Kusumoto L, M arques S, H aas VJ, Rodrigues RAP. A dultos e idosos em hemodiálise: avaliação da qualidade de vida relacionada à saúde. A cta Paul E nferm. 2008;21(n esp):152-9.

20 Romão M AF, Romão Junior JE, Belasco AGS, Barbosa DA. Qualidade de vida de pacientes com insuficiência renal crônica terminal em hemodiálise de alta eficiência. Rev G aúcha E nferm. 2006;27(4):5938.

Recebido em: 07/ 02/ 2011

A provado em: 02/ 05/ 2011 